\title{
INVESTIGACIÓN
}

\section{El aceite de oliva virgen y la distribución y movimiento transmembrana del colesterol en la membrana de eritrocito humano}

\author{
Por F. J. G. Muriana y V. Ruiz-Gutiérrez \\ Instituto de la Grasa (CSIC), Apartado 1078, 41012 - Sevilla
}

\section{RESUMEN}

\begin{abstract}
El aceite de oliva virgen y la distribución y movimiento
\end{abstract} transmembrana del colesterol en la membrana de eritrocito humano.

Se estudió el efecto de la ingesta de aceite de oliva virgen (en comparación con la ingesta de aceite de girasol alto-oleico) sobre la distribución del colesterol y su movimiento entre las monocapas externa e interna de la membrana de eritrocito de sujetos sanos y pacientes hipertensos (normo- e hipercolesterolémicos). También se determinaron la actividad del contratransporte sodio-litio en eritrocito y la presión arterial. Cada período de la intervención dietética tuvo una duración de 4 semanas, con 4 semanas de «lavado» (retorno a la dieta basal) entre las dietas monoinsaturadas. El aceite de oliva virgen (pero no el aceite de girasol alto-oleico) fue capaz de reducir la actividad anormalmente alta del contratransporte sodio-litio, de normalizar la distribución del colesterol y velocidad de movimiento transmembrana, y de reducir significativamente la presión arterial sistólica y diastólica de pacientes hipertensos. Estas experiencias parecen indicar que mediante una dieta enriquecida con aceite de oliva virgen es posible controlar y/o prevenir parcialmente la alteración de distintos parámetros de interconexión patogénica relacionados con la hipertensión arterial humana y de clara influencia sobre la Patología Cardiovascular. Estos procesos no pueden adscribirse exclusivamente al contenido de acido oleico en el aceite de oliva virgen.

PALABRAS-CLAVE: Aceite de girasol alto oleico - Aceite de oliva virgen - Contratransporte sodio-litio - Distribución del colesterol - Humanos - Movimiento transmembrana del colesterol - Presión arterial.

\section{SUMMARY}

Virgin olive oil and cholesterol distribution and transbilayer movement in human erythrocyte membrane.

The influence of virgin olive oil intake (with regard to the ingestion of high-oleic sunflower oil) on cholesterol distribution and transbilayer movement in the erythrocyte membrane of healthy subjects and hypertensive patients (with or without concomitant hypercholesterolaemia) was studied. The activity of enythrocyte sodium-lithium countertransport and blood pressure were also determined. The study was conducted over two 4-week periods, with a washout (4-week) period between both monounsaturated diets. Olive oil (but not high-oleic sunflower oil) was able to reduce the erythrocyte sodium-lithium countertransport activity, to normalize the enythrocyte membrane cholesterol distribution and transbilayer movement, and to significantly diminish the systolic and diastolic blood pressures in hypertensive patients. Our data indicate that olive oil enriched-diet may modulate and/or partially prevent the alteration of those pathogenic parameters related to human hypertension and Cardiovascular Pathology. These processes cannot be exclusively ascribed to the content of oleic acid in virgin olive oil.

KEY-WORDS: Arterial blood pressure - Cholesterol distribution - Cholesterol transbilayer - High oleic sunflower oil - Humans - Sodium-lithium countertransport - Virgin olive oil.

\section{INTRODUCCIÓN}

El concepto simple de considerar el colesterol plasmático como un punto final de referencia, aunque valioso para el diagnóstico clínico, es insuficiente. El control de este parámetro constituye un objetivo clave en la lucha para lograr la disminución de las enfermedades cardiovasculares en nuestra sociedad, pero no debe ser el único. Estudios recientes en eritrocitos humanos (Muriana, 1994) (Muriana, 1995), como modelo experimental de membrana biológica, sugieren que otros parámetros celulares pueden ser de interés además de la concentración de colesterol en el plasma sanguíneo de sujetos sanos (prevención primaria) y pacientes normo-e hipercolesterolémicos con hipertensión arterial (prevención secundaria).

Brevemente, el colesterol exógeno y endógeno se transportan en la sangre unidos a las lipoproteínas [esencialmente a las de baja densidad (LDL)]; este colesterol entra en contacto con las células mediante una interacción con la monocapa externa de la membrana (bicapa lipídica), vía receptores o no; y entonces tiene lugar un movimiento transmembrana que permite su disociación y liberación al espacio citoplasmático. Pues, bien, en este tránsito se produce un fénomeno de distribución asimétrica del colesterol [es decir, las moléculas de colesterol se localizan preferentemente en la monocapa interna de las membranas (distribución asimétrica transmembrana), formando dominios ricos y dominios pobres en cada monocapa (distribución asimétrica lateral)] y ún fénóniemo de veločidad de movimiento del colesterol de una monocapa a otra (Figura 1). Lo 
más interesante es que ambos parámetros se correlacionan perfectamente con la actividad del contratransporte sodio-litio (Muriana, 1996a,b), una proteína de membrana muy relevante en el equilibrio intracelular del ión sodio y considerada un importante marcador de la hipertensión arterial humana (Canessa, 1991).

En las membranas, los lípidos co-existen como dominios discretos; y la interpretación de esta localización física de múltiples dominios de colesterol es también dependiente de su velocidad de movimiento en la bicapa lipídica (Brasaemle, 1988). El hecho de que algunas proteínas se encuentren físicamente localizadas en dominios ricos de colesterol, en dominios pobres de colesterol, o incluso específicamente asociadas con el colesterol (Schroeder, 1991), indica que tales dominios pudieran influir sobre la actividad o función de estas proteínas. Parece ser que el mecanismo de acción se basa en la capacidad de separación de fases del colesterol que promueve la agregación y subsiguiente activación del transportador iónico o cualquier otra proteína de membrana. Tal hipótesis podría explicar la activación del contra-

\section{DISTRIBUCIÓN ASIMÉTRICA DEL COLESTEROL}

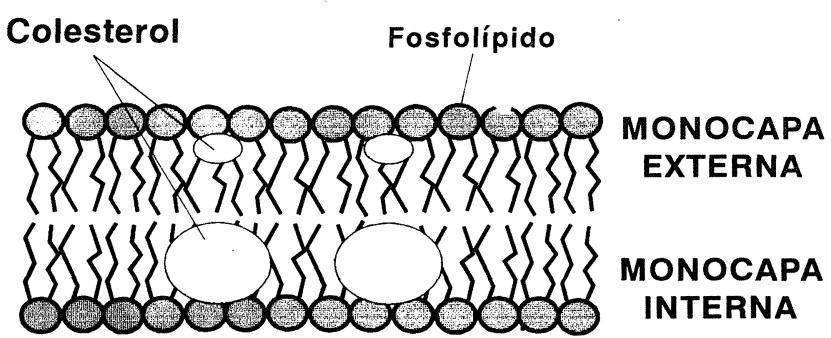

\section{MOVIMIENTO TRANSMEMBRANA DEL COLESTEROL}

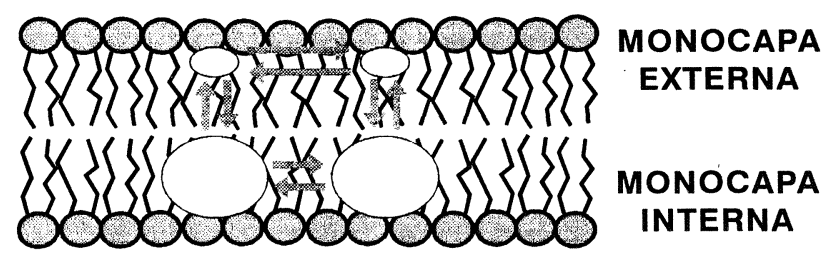

Figura 1 transporte sodio-litio en la hipertensión arterial, por cuanto que la distribución asimétrica del colesterol puede formar parte de las anomalías patogénicas asociadas con la regulación de la presión arterial (Schroeder, 1991) (Corrocher, 1992) (Engler, 1992) (Rothblat, 1992) (Straznicky, 1993). En este sentido, cabe considerar la distribución asimétrica del colesterol en las membranas celulares como el sustrato anatómico de las alteraciones de los transportadores de membrana, con implicación directa en el transporte reverso del colesterol, en la hipertensión arterial y por ende en las enfermedades cardiovasculares.

En definitiva, la distribución del colesterol entre monocapas y su velocidad de movimiento de una monocapa a otra pueden considerarse como nuevos parámetros a estudiar en la Patología Cardiovascular, ya que en pacientes de alto riesgo para padecer accidentes coronarios (hipertensos e hipercolesterolémicos) participan de una correlación estricta con los valores de presión arterial y colesterolemia (Muriana, 1996a,b). El contenido de colesterol ligado a la cara interna de la membrana de eritrocito parece estar aumentado en pacientes hipertensos (Muriana, 1994) y relacionado con la modificación de la viscosidad celular en la hipertensión arterial (Schroeder, 1991) (Ronquist, 1992) (Liscum, 1994). Además, los dominios discretos de colesterol en las membranas y su movilidad transmembrana pueden producir cambios en la funcionalidad del endotelio vascular, la regulación del tono vascular, la permeabilidad endotelial y otros mecanismos asociados a alteraciones hemodinámicas importantes que repercutan en el inicio y desarrollo de enfermedades cardiovasculares (Liscum, 1994) (Schroeder, 1991) (Straznicky, 1993) (Tamargo, 1993).

La ingestà de aceite de oliva virgen puede modificar el perfil lipídico en el plasma sanguíneo y los lípidos de membrana en sujetos sanos y pacientes hipertensos (Ruiz-Gutiérrez, 1997). Sin embargo, no existen en la actualidad estudios sobre los efectos del aceite de oliva virgen en la distribución asimétrica del colesterol y su movimiento transmembrana. Si este aceite modificara estos parámetros, sería una novedad absoluta en el campo científico de la alimentación relacionado con las enfermedades cardiovasculares, especialmente porque se establecería, por vez primera, un nexo de unión entre enfermedades metabólicas (hipertensión arterial, dislipoproteinemias) y las membranopatías relacionadas con la localización física y movilidad del colesterol en las células. La coordinación de estos factores de riesgo y estos nuevos parámetros en la Patología Cardiovascular puede así contribuir a un mejor conocimiento de los aspectos nutritivos, procesos metabólicos y papel en distintas enfermedades del aceite de oliva virgen.

Más aún, con estas medidas «no farmacológicas" pueden evitarse los efectos secundarios deri- 
vados de los fármacos hipotensores, de importante repercusión nutricional como intolerancia a los carbohidratos, aumento de los niveles plasmáticos de ácido úrico y triglicéridos, modificación de los niveles plasmáticos de calcio, potasio y sodio, e incluso alcalosis metabólica (Otero, 1990). Estos hallazgos parecen sustentarse en ciertos factores comunes que actúan, en general de manera combinada, sobre las membranas del árbol vascular (Tamargo, 1993). También ha de considerarse que, cuando coexisten hipertensión arterial e hipercolesterolemia, aumenta de manera profunda el riesgo de complicaciones cardiovasculares arterioscleróticas (Figura 2).

Dada la importancia del consumo del aceite de oliva virgen en nuestro país y considerando su trascendental papel en la prevención y tratamiento de las enfermedades cardiovasculares, nos ha parecido interesante estudiar el efecto del aceite de oliva virgen de manera comparativa con otra fuente vegetal de ácidos grasos monoinsaturados, como es el aceite de girasol alto-oleico. En este estudio han participado sujetos sanos y pacientes con factores de riesgo

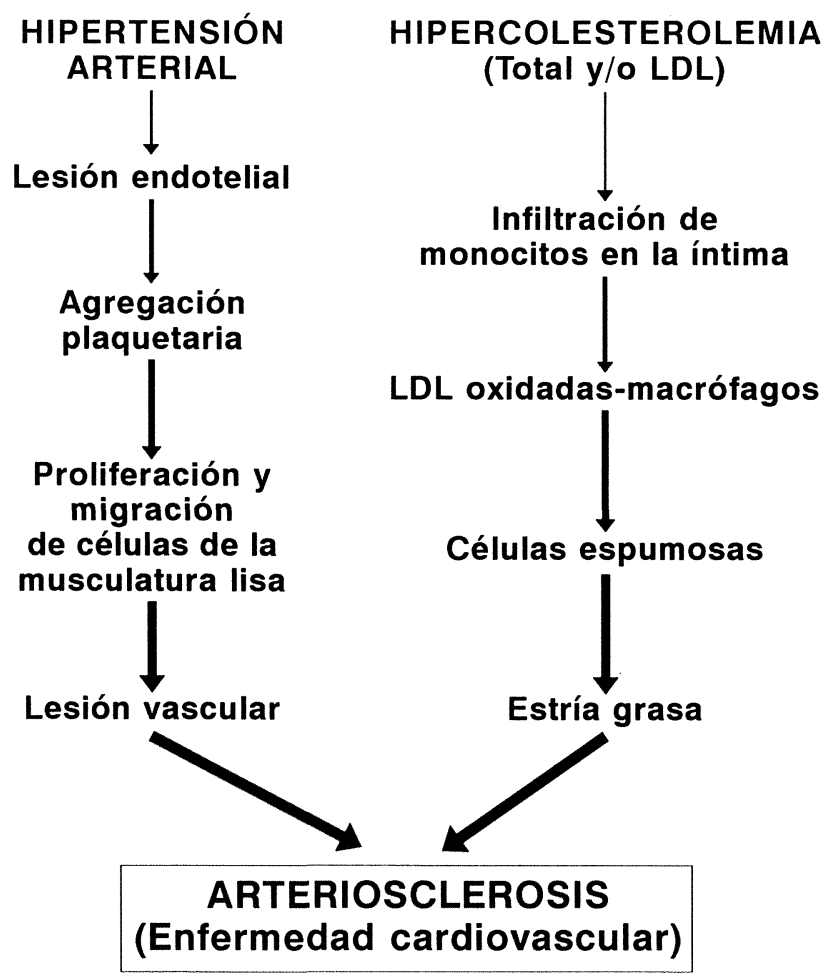

Figura 2 como la hipertensión arterial e hipercolesterolemia (tres grupos experimentales: sujetos sanos, pacientes normocolesterolémicos e hipertensos, y pacientes hipercolesterolémicos e hipertensos). Los objetivos principales han sido determinar la influencia de la dieta sobre la actividad del contratransporte sodio-litio, distribución y movimiento transmembrana del colesterol en eritrocito, y presión arterial (sistólica y diastólica). Mediante el presente estudio esperamos, además, comprobar la hipótesis de la implicación de la distribución asimétrica del colesterol y el transporte de éste en las membranas celulares como nuevos factores de riesgo cardiovascular modificables mediante la dieta y asociados a la patogénesis de la hipertensión arterial humana.

\section{PACIENTES, MATERIAL Y MÉTODOS}

\section{Población, dieta y diseño experimental}

La población estuvo constituida por 28 mujeres divididas en un grupo de mujeres sanas $(n=12)$ ( $54 \pm 3$ años), otro de pacientes hipertensas y normocolesterolémicas $(n=8)\left(55_{ \pm} 4\right.$ años), y otro de pacientes hipertensas e hipercolesterolémicas $(n=8)$ ( $56 \pm 3$ años). Un mes antes y durante la experiencia, ninguna estuvo sometida a tratamiento farmacológico conocido que afectara a la presión arterial. El criterio de hipertensión fue una presión sistólica $\geq 140$ $\mathrm{mm} \mathrm{Hg}$ y una presión diastólica $\geq 90 \mathrm{~mm} \mathrm{Hg}$, y el criterio de hipercolesterolemia fue una concentración en plasma de colesterol total $\geq 6,22 \mathrm{mmol} / \mathrm{L}$ y $\mathrm{LDL}$ colesterol $\geq 4,14 \mathrm{mmol} / \mathrm{L}$ después de 12 horas de ayuno.

La dieta consistió en alimentos ordinarios distribuidos en menús de una semana cada uno. La dieta basal fue similar a la recomendada por el Programa Nacional (estadounidense) de Educación sobre el Colesterol. Desde ese momento se inició la intervención dietética, siendo la principal diferencia entre la.dieta basal, la dieta enriquecida con aceite de oliva virgen y la dieta enriquecida con aceite de girasol alto-oleico, la fuente de energía de origen graso. El contenido calórico de la dieta base, así como el porcentaje de carbohidratos y proteínas, se mantuvo constante con respecto a las dietas suplementadas con aceite de oliva virgen o aceite de girasol alto-oleico.

El diseño experimental contemplaba dos fases: la primera de análisis de la dieta basal durante 3 semanas y la segunda de intervención dietética con dos aceites ricos en ácidos grasos monoinsaturados (aceite de girasol alto-oleico y aceite de oliva virgen, por este orden) durante 8 semanas de tratamiento (4 semanas con cada dieta), con un período de lavado conveniente (mínimo 4 semanas de retorno a los criterios de la dieta basal). 
Más detalles sobre la población, dieta y diseño experimental se citan en el trabajo de Ruiz-Gutiérrez (1997).

\section{Actividad del transportador (contratransporte) sodio-litio en eritrocito}

\section{Aislamiento de eritrocitos}

Mediante punción en la vena antecubital, se recogieron muestras de sangre en tubos heparinizados (Vacutainer $($ ). Los eritrocitos se obtuvieron mediante centrifugación a $800 \times \mathrm{g}$, durante 10 minutos a $4^{\circ} \mathrm{C}$, aspirándose y eliminándose el sobrenadante y la capa de leucocitos y plaquetas. Se lavaron cuatro veces con una solución que contenía $\mathrm{MgCl}_{2} 110$ $\mathrm{mmol} / \mathrm{L}$ y se resuspendieron en una solución amortiguadora con sobrecarga de $\mathrm{Li}^{+}$(«medio de sobrecarga") $\left(\mathrm{Li}_{2} \mathrm{CO}_{3} 75 \mathrm{mmol} / \mathrm{L}\right.$, glucosa $10 \mathrm{mmol} / \mathrm{L}$, MOPS-Tris $75 \mathrm{mmol} / \mathrm{L}, \mathrm{pH} 7.4$ ) hasta un hematocrito del $20 \%$.

\section{Metodología}

La técnica empleada para la determinación de la velocidad máxima del contratransporte sodio-litio fue la de Canessa (1988); según la cual, el flujo de $\mathrm{Na}^{+}$ dependiente de este sistema se considera como el componente de la salida neta de $\mathrm{Na}^{+}$que, siendo resistente a la ouabaína y a la bumetanida, es estimulado por $\mathrm{Li}^{+}$. Brevemente, las células resuspendidas en el medio de sobrecarga se incubaron durante 60 minutos a $37^{\circ} \mathrm{C}$, tiempo al cabo del cual se realizaron cuatro lavados consecutivos con solución de $\mathrm{MgCl}_{2} 115 \mathrm{mmol} / \mathrm{L}$. Seguidamente, los eritrocitos fueron centrifugados a $3500 \times \mathrm{g}$, durante 2 minutos a $4^{\circ} \mathrm{C}$. Se tomaron 4 alícuotas para determinar los eflujos de $\mathrm{Li}^{+}$en presencia o ausencia de $\mathrm{Na}^{+}$, la concentración de $\mathrm{Li}^{+}$intracelular y el hematocrito.

Los eflujos de $\mathrm{Li}^{+}$se determinaron a partir de la incubación de alícuotas $(1 \mathrm{~mL})$ de la suspensión celular con solución amortiguadora de $\mathrm{NaCl}(\mathrm{NaCl} 150$ $\mathrm{mmol} / \mathrm{L}$, glucosa $10 \mathrm{mmol} / \mathrm{L}$, ouabaína $0,1 \mathrm{mmol} / \mathrm{L}$, MOPS-Tris $10 \mathrm{mmol} / \mathrm{L}, \mathrm{pH} 7.4$ ), durante 60 minutos a $4^{\circ} \mathrm{C}$; o bien con solución amortiguadora carente de $\mathrm{NaCl}\left(\mathrm{MgCl}_{2} 75 \mathrm{mmol} / \mathrm{L}\right.$, glucosa $10 \mathrm{mmol} / \mathrm{L}$, ouabaína $0,1 \mathrm{mmol} / \mathrm{L}$, sacarosa $85 \mathrm{mmol} / \mathrm{L}$, MOPS-Tris 10 $\mathrm{mmol} / \mathrm{L}, \mathrm{pH} 7.4$ ), durante el mismo tiempo y temperatura. Los eritrocitos se centrifugaron entonces a $3500 \times \mathrm{g}$, durante 2 minutos a $4^{\circ} \mathrm{C}$, y la concentración de $\mathrm{Li}^{+}$se determinó en los sobrenadantes mediante espectrofotometría de absorción atómica (Perkin-Elmer 460). La velocidad máxima del contratransporte se determinó a partir de la diferencia de concentración de $\mathrm{Li}^{+}$en los sobrenadantes antes mencionados, considerando que el sistema actúa bajo condiciones de saturación, y se expresó en $\mathrm{mmol} \times$ [hora $x$ litro células] ${ }^{-1}$. En los casos en los que la hemolisis fue apreciable, se corrigió la concentración de $\mathrm{Li}^{+}$midiendo la hemoglobina, calculando el $\mathrm{Li}^{+}$presente en el sobrenadante secundario a la hemolisis eritrocitaria y restando la correspondiente concentración de $\mathrm{Li}^{+}$.

\section{Distribución y transporte transmembrana del colesterol en eritrocito}

\section{Aislamiento de eritrocitos}

Mediante punción en la vena antecubital, se recogieron muestras de sangre en citrato sódico al $0,38 \%$. Los eritrocitos se obtuvieron mediante centrifugación a $1750 \times g$, durante 5 minutos a $4^{\circ} \mathrm{C}$, aspirándose y eliminándose el sobrenadante y la capa de leucocitos y plaquetas. Se lavaron tres veces con solución amortiguadora salina («medio salino») ( $\mathrm{NaCl} 145 \mathrm{mmol} / \mathrm{L}, \mathrm{KCl} 5 \mathrm{mmolL}$ y fosfato sódico 5 $\mathrm{mmol} / \mathrm{L}, \mathrm{pH}$ 7.4) y tres veces con solución amortiguadora de ensayo («medio de ensayo») (sacarosa $310 \mathrm{mmol} / \mathrm{L}, \mathrm{MgSO}_{4} 1 \mathrm{mmol} / \mathrm{L}$ y fosfato sódico 5 $\mathrm{mmol} / \mathrm{L}, \mathrm{pH}$ 7.4), donde se resuspendieron hasta un hematocrito del $3 \%$ (aprox. $50 \mu \mathrm{g}$ de colesterol de eritrocito/mL de suspensión celular). Los estudios se realizaron en las primeras seis horas tras la obtención de los eritrocitos.

\section{Marcaje isotópico de eritrocitos}

Alícuotas de $3 \mathrm{~mL}$ de la suspensión celular se incubaron a $4{ }^{\circ} \mathrm{C}$, con $185 \times 10^{6} \mathrm{~Bq}\left[{ }^{3} \mathrm{H}\right]$-colesterol $\left(1.88 \times 10^{12} \mathrm{~Bq} / \mathrm{mmol}\right)$ en $50 \mu \mathrm{L}$ de etanol, durante cinco minutos. De este modo se permitió el marcaje de la monocapa externa de la membrana del eritrocito. Las células marcadas se lavaron 4 veces en medio salino y 3 veces en medio de ensayo a $4{ }^{\circ} \mathrm{C}$, realizando la última centrifugación a $12000 \times$ g, durante 30 segundos.

\section{Tratamiento con colesterol oxidasa}

La enzima colesterol oxidasa se empleó para oxidar a colestenona el colesterol de la monocapa externa de la membrana de eritrocito, método mediante el cual se determinan sus cinéticas de oxidación y movimiento transmembrana (Brasaemle, 1988). Para ello, dependiendo del experimento, los eritrocitos se preincubaron a $37^{\circ} \mathrm{C}$ (entre 0 y 100 min) y se trataron brevemente con colesterol oxidasa (procedente de Brevibacterium sp., $6 \mathrm{U} / \mathrm{mL}$ ) durante $20 \mathrm{~s}$, o se preincubaron durante $15 \mathrm{~s}$, a $37^{\circ} \mathrm{C}$, 
y entonces se trataron de manera continua con la enzima (entre 0 y 25 minutos). Se tomaron alícuotas de $0,25 \mathrm{~mL}$ según el tiempo indicado en cada figura o tabla, que se sometieron a extracción con $1 \mathrm{~mL}$ de cloroformo-metanol (2:1, vol:vol). La fase orgánica se dividió en dos porciones iguales y se utilizó para el análisis de la masa y radioactividad de los esteroles (colesterol y colestenona).

\section{Determinación de la masa y radioactividad de es- teroles}

La determinación de la masa del colesterol y la colestenona se llevó a cabo mediante cromatografía de gases, con columna de separación de esteroles. Para ello, se utilizó un cromatógrafo de gases Hewlett-Packard 5790A, equipado con un integrador Hewlett-Packard 3390a. Las muestras se redisolvieron en $15 \mu \mathrm{L}$ de éter isopropílico frío y se inyectaron aproximadamente $3 \mu \mathrm{L}$ en una columna Supelcoport $80 / 100$ (3\% OV-17; Teknokroma 14280C). El gas portador fue nitrógeno $\left(20 \mathrm{~cm}^{3} / \mathrm{min}\right)$. La temperatura de la columna fue $325^{\circ} \mathrm{C}$, mientras que las del inyector y detector fueron 350 y $360^{\circ} \mathrm{C}$, respectivamente. La masa de cada esterol se determinó comparando las áreas de sus picos con el área del pico de un patrón interno (estigmasta-4,22-dienona) de concentración conocida.

La cuantificación de la radioactividad del colesterol y la colestenona se realizó mediante cromatografía en capa fina. Los extractos lipídicos se redisolvieron en cloroformo $(20 \mu \mathrm{L})$ y se dispusieron en placas de sílica gel 60G (0,25 mm de espesor; Kieselgel 60G). El desarrollo de las placas se llevó a cabo en hexanoéter etílico-ácido acético (80:25:1, vol:vol:vol). La radiación se detectó con la ayuda de un analizador lineal semi-automático Berthold, conectado a un ordenador Apple lle provisto con un software de trabajo individual. Las bandas radiactivas se rasparon de las placas, se colocaron en viales de centelleo y, tras añadir $10 \mathrm{~mL}$ de líquido de centelleo, se determinaron las dpm en un contador Beckman calibrado.

\section{Estabilidad del eritrocito al tratamiento con coles- terol oxidasa}

La integridad de los eritrocitos tratados con colesterol oxidasa se evaluó mediante la medida de la absorbancia a 540 y 410 (banda de Soret) $\mathrm{nm}$ del sobrenadante después del centrifugado, a $12000 \times g$ durante $30 \mathrm{~s}$, de alícuotas de la mezcla. La absorción de la hemoglobina a estas longitudes de onda fue comparada con la absorbancia del sobrenadante de una cantidad equivalente de células lisadas en una solución amortiguadora hipotónica (fosfato sódico $5 \mathrm{mM}, \mathrm{NaCl} 17 \mathrm{mmol}$, pH 7.4) durante 75 minutos a temperatura ambiente.

También se midieron el volumen corpuscular medio (MCV) (Technicon $\mathrm{H}-1$ blood cell counter) de los eritrocitos tratados y la actividad de las enzimas lác- tico deshidrogenasa y adenilato quinasa, como marcadoras de la rotura celular, al ser enzimas eminentemente citosólicas (Brolin, 1983) (Vassault, 1983).

Estos ensayos se realizaron en presencia (sacarosa $310 \mathrm{mmol} / \mathrm{L}$, fosfato sódico $5 \mathrm{mmol} / \mathrm{L}, \mathrm{MgSO}_{4} 1$ $\mathrm{mmol} / \mathrm{L}, \mathrm{pH}$ 7.4) y en ausencia de iones magnesio (sacarosa $310 \mathrm{mmol} / \mathrm{L}$, fosfato sódico $5 \mathrm{mmol} / \mathrm{L}, \mathrm{pH} 7.4$ ); y en presencia de iones magnesio junto a glucosa (glucosa $10 \mathrm{mmol} / \mathrm{L}$, sacarosa $300 \mathrm{mmol} / \mathrm{L}$, fosfato sódico $5 \mathrm{mmol} / \mathrm{L}, \mathrm{MgSO}_{4} 1 \mathrm{mmol} / \mathrm{L}, \mathrm{pH} 7.4$ ), para determinar el efecto del "ayuno" sobre los eritrocitos durante el tratamiento con la colesterol oxidasa.

\section{Medidas de presión arterial}

Se realizaron dos tomas de presión arterial con esfigmomanómetros de mercurio y manguitos de dimensiones adecuadas al tamaño del brazo, siguiendo las recomendaciones de la OMS. Ambas medidas fueron en posición de sentado, la primera a los 5 minutos y la segunda a los 10-15 minutos de iniciar la entrevista. Se definió la presión arterial sistólica y diastólica, respectivamente, el primer y quinto ruidos de Kórotkotf, y la resultante de la media de las dos lecturas. La definición de hipertensión fue considerada según el Comité Asesor de Consenso para el control de la Hipertensión Arterial en España (Aranda, 1993).

\section{Análisis estadístico}

Para el análisis estadístico de los datos se calcularon las medias con sus desviaciones estándares. Las diferencias entre las dietas enriquecidas con aceite de oliva virgen y aceite de girasol alto-oleico se analizaron como medidas repetitivas de análisis de varianza (ANOVA), aplicando la correccción de Tukey (Honestly Significant Difference T-Method) como método de comparación post-hoc (nivel de significancia: $5 \%$ ). Las correlaciones se determinaron empleando el test de regresión lineal de Pearson, calculando también los intervalos con un $95 \%$ de confianza para los coeficientes de regresión. Estos estudios se realizaron con los paquetes estadísticos GraphPAD InStat (GraphPAD Software) y CoStat (CoHort Software).

\section{RESULTADOS Y DISCUSIÓN}

\section{Actividad del contratransporte sodio-litio en eritrocito}

Entre las anomalías del metabolismo celular del ión sodio en pacientes hipertensos, con respecto a su significado fisiopatológico, un aumento en la actividad (velocidad máxima) del contratransporte sodio-litio se considera una "anomalía estable de translocación" (Coca, 1993). Esta proteína de mem- 
brana está muy relacionada con el intercambiador sodio-protón y el intercambiador cloruro/bicarbonato, sistemas por otra parte implicados en la regulación del pH intracelular (Blaustein, 1984). Cuando fallan estos sistemas de control iónico, se produce además un aumento de la reabsorción tubular de $\mathrm{Na}^{+}$y de la concentración del $\mathrm{Ca}^{2+}$ libre citosólico (Aviv, 1988a,b). Por estos motivos, entre otros, la hipertensión arterial asociada al aumento de la actividad del contratransporte sodio-litio es considerada uno de los principales factores de riesgo en las enfermedades cardiovasculares. En estas condiciones, aumenta el tono y la reactividad vascular, y se produce una hipertrofia-hiperplasia vascular (proliferación y migración de las células de la musculatura lisa hacia el espacio subintimal, con cambios en el fenotipo de contráctil a sintético) (Tamargo, 1993).

La actividad de este contratransporte fue significativamente superior en pacientes hipertensas normocolesterolémicas $(22 \%, p<0.01) y$, sobre todo, en pacientes hipertensas hipercolesterolémicas $(57 \%$, $\mathrm{p}<0.01$ ) que en mujeres sanas (Tabla 1). Con respecto a la dieta basal, tras la ingesta de aceite de oliva virgen, los eritrocitos de mujeres sanas no modificaron su capacidad intercambiadora de $\mathrm{Na}^{+}$por $\mathrm{Li}^{+}$. Sin embargo, las actividades anormalmente altas del contratransporte sodio-litio en el eritrocito de pacientes hipertensas fueron significativamente reducidas hasta valores próximos al del grupo control con la dieta basal. Sorprendentemente, la ingesta de aceite de girasol alto-oleico afectó negativamente la capacidad funcional de este contratransporte, aumentando drásticamente su actividad en los tres grupos experimentales, incluyendo los eritrocitos de mujeres sanas. Por lo tanto, el aceite de oliva virgen (y no el aceite de girasol alto-oleico) produce cambios en las membranas celulares que contribuyen a normalizar la actividad del contratransporte sodio-litio (anormalmente alta) en pacientes hipertensos.

\section{Distribución del colesterol en la membrana de eritrocito}

La colesterol oxidasa de Brevibacterium sp. se utilizó para oxidar el colesterol en la monocapa externa de la bicapa lipídica, que tiene lugar en el grupo $\beta$-hidroxilo de la posición 3 y produce $\Delta^{4}$-colestenona (Lange, 1992). La presencia de iones magnesio en las soluciones amortiguadoras de lavado y ensayo fue suficiente para mantener la integridad de los eritrocitos (indispensable para asegurar que la colesterol oxidasa no tuviera acceso a la monocapa interna del eritrocito) (Muriana, 1996c). No hubo hemólisis ni liberación de láctico deshidrogenasa ni adenilato quinasa significativas al medio extracelular tras el tratamiento continuo con colesterol oxidasa al final del tiempo más largo de incubación. Tampoco fue imprescindible la presencia de glucosa.

Se construyeron gráficas de oxidación del colesterol con respecto al tiempo de incubación, tanto con eritrocitos de mujeres sanas como de pacientes hipertensas, antes y después de la ingesta de los aceites monoinsaturados (Figura 3 ). Con respecto a la dieta basal, los tiempos medios de oxidación (cuando se alcanza el $50 \%$ de oxidación del total de colesterol) fueron superiores en los eritrocitos de pacientes hipertensas normocolesterolémicas $(38 \%, \mathrm{p}<0.001)$ y pacientes hipertensas hipercolesterolémicas $(57 \%, p<0.001)$ que en mujeres sanas (Tabla 2); no siendo significativas las diferencias entre ambos grupos de pacientes hipertensas. Estos resultados, junto con la rápida oxidación del colesterol $(15.2 \pm 1.9 \%$ para los sujetos sanos; $8.2 \pm 1.2 \%$ para los pacientes hipertensos normocolesterolémicas; $y$ 7.4 $1.3 \%$ para los pacientes hipertensos hipercolesterolémicas) de la monocapa externa al inicio del tratamiento con colesterol oxidasa, sugieren que el colesterol se distribuye asimétricamente en la bicapa lipídica del eritrocito. Es decir, existe más colesterol en la monocapa interna que en la externa, en concordancia con lo descrito por otros autores (Brasaemle, 1988) (Liscum, 1994) (Schroeder, 1991) (Straznicky, 1993).

Tabla 1

Actividad del contratransporte sodio-litio en eritrocito humano durante la dieta basal y tras las dietas enriquecidas con aceite de oliva virgen y aceite de girasol alto-oleico

\begin{tabular}{|c|c|c|c|}
\hline$\checkmark$ Población estudiada & BASAL & $\begin{array}{c}\text { ACEITE DE } \\
\text { OLIVA } \\
\text { VIRGEN }\end{array}$ & $\begin{array}{l}\text { ACEITE DE } \\
\text { GIRASOL } \\
\text { ALTO OLEICO }\end{array}$ \\
\hline Mujeres sanas & $0,258 \pm 0,076$ & $0,248 \pm 0,037$ & $0,299 \pm 0,104$ \\
\hline HT/normocolesterolémicas & $0,314 \pm 0,043$ & $0,266 \pm 0,032^{*}$ & $0,355 \pm 0,086^{*}$ \\
\hline HT/hipercolesterolémicas & $0,405 \pm 0,086$ & $0,334 \pm 0,061^{\star}$ & $0,540 \pm 0,098^{*}$ \\
\hline
\end{tabular}

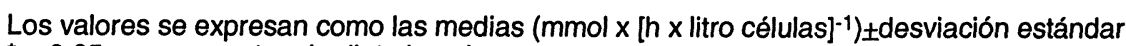

${ }^{\star} \mathrm{p}<0,05$, con respecto a la dieta basal 


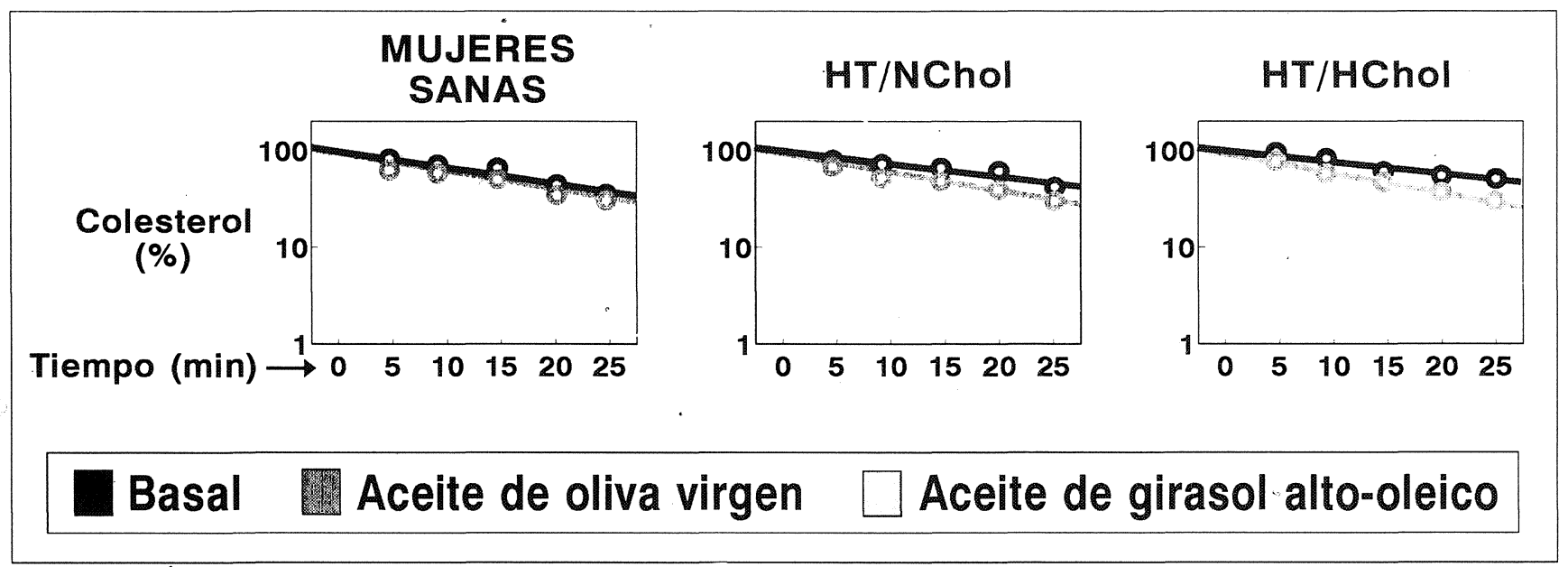

Figura 3

Tabla 2

Tiempos medios de oxidación del colesterol en eritrocito humano durante la dieta basal y tras las dietas enriquecidas con aceite de oliva virgen y aceite de girasol alto-oleico

\begin{tabular}{|c|c|c|c|}
\hline$\checkmark$ Población estudiada & BASAL & $\begin{array}{l}\text { ACEITE DE } \\
\text { OLIVA } \\
\text { VIRGEN }\end{array}$ & $\begin{array}{c}\text { ACEITE DE } \\
\text { GIRASOL } \\
\text { ALTO OLEICO }\end{array}$ \\
\hline Mujeres sanas & $14,9 \pm 2,6$ & $15,0 \pm 3,1$ & $4,2 \pm 0,8^{\star}$ \\
\hline HT/normocolesterolémicas & $20,6 \pm 2,8$ & $14,8 \pm 2,5^{*}$ & $4,9 \pm 1,7^{\star}$ \\
\hline HT/hipercolesterolémicas & $23,4 \pm 4,2$ & $15,6 \pm 3,9^{*}$ & $5,5 \pm 0,6^{\star}$ \\
\hline
\end{tabular}

Los valores se expresan como las medias (minutos) \pm desviación estándar ${ }^{*} \mathrm{p}<0,001$, con respecto a la dieta basal

La difusión del colesterol entre monocapas fue más lenta en el eritrocito de pacientes hipertensas, lo cual se asocia a la presencia de dominios ricos en colesterol localizados en la monocapa interna (Liscum, 1994) (Schroeder, 1991). Los lípidos en un dominio rico en colesterol están fuertemente empaquetados, son menos proclives a la interacción con proteínas e incluso dificultan la translocación de las mismas.

Tras la ingesta de aceite de oliva virgen con respecto a la dieta basal, los tiempos medios requeridos para la oxidación del colesterol fueron reducidos hasta su normalización en los eritrocitos de pacientes hipertensas (Tabla 2) y la distribución asimétrica del colesterol entre monocápas también fue normalizada (Figura 4). Estas interesantes observaciones pueden relacionarse con la incorporación de los ácidos grasos de la familia $n-3$ en la membrana (RuizGutiérrez, 1997). Según Knapp (1994), los ácidos docosapentaenoico (22:5n-3) y docosahexaenoico $(22: 6 n-3)$ se incorporan de manera selectiva en los fosfolípidos localizados en la monocapa interna de la membrana de eritrocito humano; estos ácidos grasos son los que precisamente aumentan su concentración en la membrana de eritrocito de pacientes hipertensas alimentadas con una dieta rica en aceite de oliva virgen. Lo más interesante es que el aceite de girasol alto-oleico no es capaz de normalizar los 
tiempos medios de oxidación del colesterol, ni su distribución asimétrica, ni aumentar el contenido en ácidos grasos de la familia n-3 en la membrana de eritrocito de pacientes hipertensas.

Mediante cálculos de dinámica molecular, otros autores (Applegate, 1986) han demostrado que, al menos, el ácido docosahexaenoico puede adoptar una disposición única ordenada en paquetes apretados (dos conformaciones espaciales posibles) que excluyen las moléculas de colesterol de la monocapa interna hacia la monocapa externa en las membranas celulares mediante una asociación negativa. Este mecanismo puede claramente explicar el efecto espectacular del aceite de oliva virgen sobre la redistribución del colesterol entre ambas monocapas en el eritrocito de pacientes hipertensas (normo- e hipercolesterolémicas).

Otro resultado interesante fue la existencia de una correlación significativa y positiva entre los tiempos medios de oxidación del colesterol y la actividad del contratransporte sodio-litio en eritrocitos de mujeres sanas (Figura $5 A$ ), de pacientes hipertensas normocolesterolémicas (Figura $5 B$ ) y de pacientes hipertensas hipercolesterolémicas (Figura $5 C$ ), con las tres dietas experimentales. Es decir, a medida que aumenta el tiempo que necesita la colesterol oxidasa para oxidar el $50 \%$ del colesterol a colestenona, entonces aumenta la velocidad máxima de intercambio entre $\mathrm{Na}^{+}$y $\mathrm{Li}^{+}$. Esta resistencia a la oxidación del colesterol en la membrana de eritrocito de pacientes hipertensas es característica de la presencia, ya confirmada, del escaso contenido de colesterol en la monocapa externa y su distribución lateral en dominios pobres; lo contrario que ocurre en la monocapa interna. La normalización de estos parámetros tras la ingesta de aceite de oliva virgen puede ser, por tanto, la causa de la tendencia a la normalización de la actividad del contratransporte sodio-litio (Schroeder, 1991) (Shouffani, 1990). La cuestión es si los dominios catalítico(s) y/o regulador(es) del intercambiador son dependientes del colesterol de la monocapa interna.

La valoración de estos resultados es de gran importancia si consideramos la distribución del colesterol entre monocapas un parámetro patogénico en el inicio y/o desarrollo de las enfermedades cardiovasculares, y su asociación con otros factores de riesgo modificables mediante la dieta.

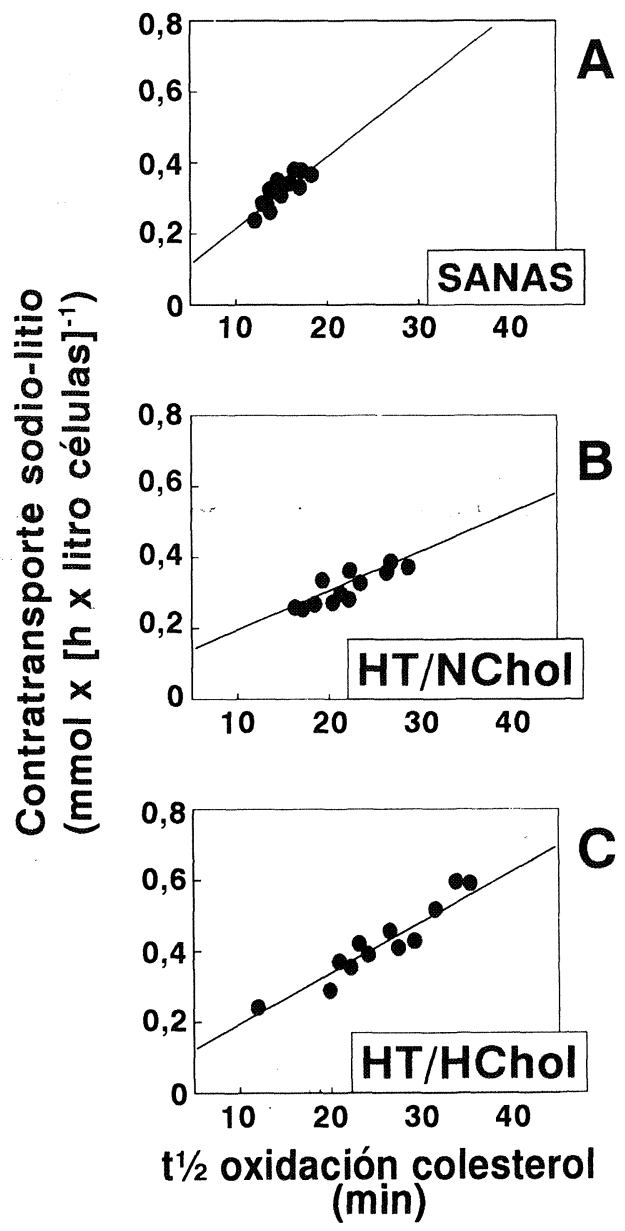

Figura 5

Figura 4 


\section{Velocidad de movimiento del colesterol en la membrana de eritrocito}

Cuando eritrocitos intactos se incuban con $\left[{ }^{3} \mathrm{H}\right]-\mathrm{co}-$ lesterol a $4^{\circ} \mathrm{C}$ se produce el marcaje isotópico de la monocapa externa. La incubación subsiguiente a 37 ${ }^{\circ} \mathrm{C}$ inicia el equilibrio del colesterol entre la monocapa externa e interna mediante un movimiento transmembrana (Brasaemle, 1988). El tratamiento con colesterol oxidasa de las células marcadas causa un descenso en la radioactividad específica $\left(\mathrm{dpm}\left[{ }^{3} \mathrm{H}\right]\right.$-esterol/ $\mu \mathrm{g}$ esterol) de la colestenona con respecto al tiempo, representando la mezcla del «pool» de colesterol radioactivo de la monocapa externa con el "pool» de colesterol no radioactivo, presumiblemente, de la monocapa interna. Esto representa la velocidad de movimiento transmembrana del colesterol.

Se construyeron gráficas de radioactividad específica de la colestenona con respecto al tiempo de incubación, tanto con eritrocitos de mujeres sanas como de pacientes hipertensas, antes y después de la ingesta de los aceites monoinsaturados (Figura 6). Con respecto a la dieta basal, los tiempos medios de radioactividad específica (cuando se alcanza el $50 \%$ del valor inicial) fueron superiores en los eritrocitos de pacientes hipertensas normocolesterolémicas $(32 \%, p<0.01)$ y de pacientes hipertensas hipercolesterolémicas $(46 \%$, $p<0.01$ ) que en mujeres sanas (Tabla 3).

Un aumento del tiempo medio de radioactividad específica de la colestenona implica una menor velocidad de movimiento del colesterol entre monocapas (Brasaemle, 1988). Esta importante reducción en la capacidad de transporte del colesterol en el eritrocito de pacientes con hipertensión arterial puede contribuir aún más a la asimetría transmembrana y lateral del colesterol, ya que la segregación física del colesterol en dominios ricos y pobres es muy dependiente de su velocidad de intercambio en la bicapa lípidica (Schroeder, 1991).

Aunque la relación exacta entre los dominios estructurales y cinéticos de colesterol en las membranas biológicas no se conoce, parece que existen diferentes "pools" cinéticos de colesterol que se denominan «pool de colesterol intercambiable» $y$ «pool de colesterol no-intercambiable" y se asocian a los dominios pobres en colesterol y a los dominios ricos en colesterol, respectivamente (Nemecz, 1988a,b) (Schroeder, 1990) (Rothblat, 1992). Estos «pools» cinéticos son generalmente el resultado de la segregación del colesterol en la bicapa lipídica (Straznicky, 1993) y, por lo tanto, no sorprende que ello se relacione directamente con los mecanismos de deabsorción del colesterol de la membrana y su intercambio espontáneo (Liscum, 1994) (Rothblat, 1992) (Schroeder, 1991). Según este modelo, puede considerarse que el colesterol de la monocapa externa de la membrana de eritrocito de pacientes hipertensos está constituido esencialmente por un «pool no-intercambiable», puesto que el colesterol difícilmente difunde a través de esta monocapa a la interna, a pesar de estar constituido por dominios pobres en colesterol.

Tras la ingesta de aceite de oliva virgen con respecto a la dieta basal, los valores de los tiempos medios de radioactividad específica de la colestenona fueron reducidos hasta casi su normalización en los eritrocitos de pacientes hipertensas (Tabla 3 ). Además, se observó una correlación significativa y negativa con la actividad del contratransporte sodio-litio en eritrocitos de mujeres sanas (Figura 7A), de pacientes hipertensas normocolesterolémicas (Figura 7B) y de pacientes hipertensas hipercolesterolémicas (Figura 7C). Es decir, cuanto mayor es la velocidad de movimiento del colesterol de una monopaca a otra, mayor es la velocidad máxima de intercambio entre $\mathrm{Na}^{+}$y $\mathrm{Li}^{+}$. Lo cual probablemente significa que interacciones lipído-lípido débiles en la membrana"celular (aumentan la capacidad de difusión de las moléculas de colesterol) pueden facilitar el flujo iónico transmembrana. El aceite de girasol alto-oleico no fue capaz de normalizar los tiempos medios de radioactividad específica de la colestenona (movimiento transmembrana del colesterol) en la membrana de eritrocito de pacientes hipertensas.

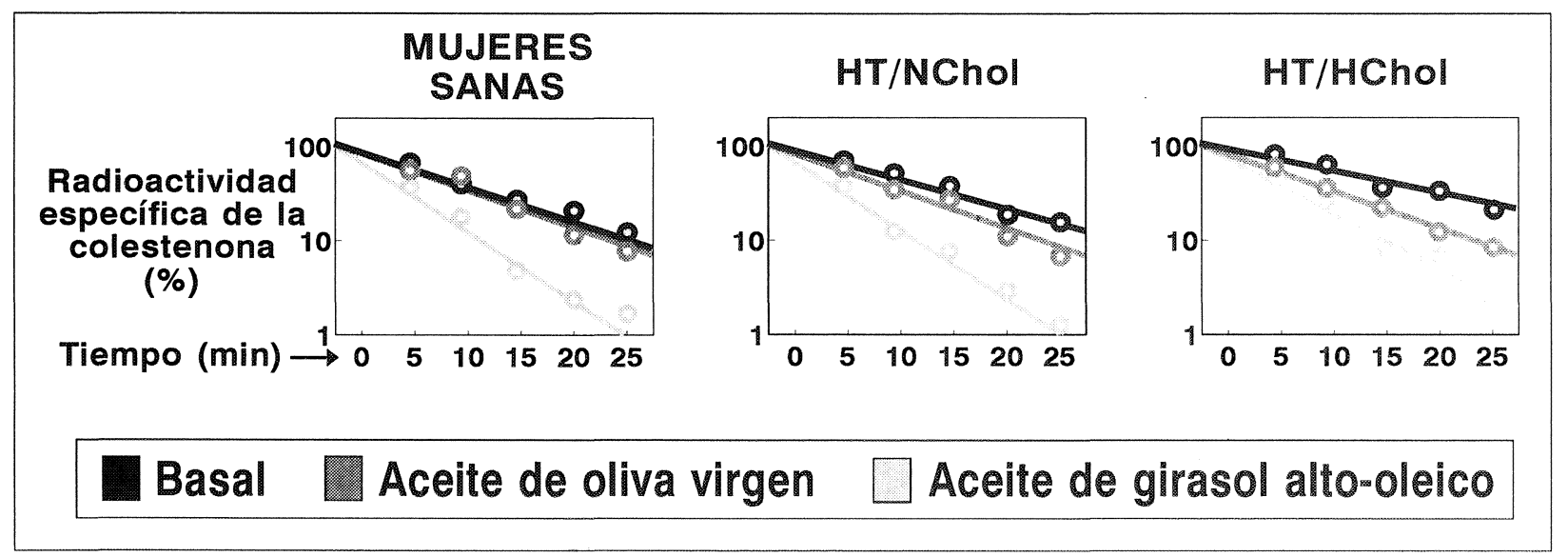

Figura 6 
Tabla 3

Tiempos medios para el descenso de la radioactividad específica de colestenona en eritrocito humano durante la dieta basal y tras las dietas enriquecidas con aceite de oliva virgen y aceite de girasol alto-oleico

\begin{tabular}{|c|c|c|c|}
\hline$\checkmark$ Población estudiada & BASAL & $\begin{array}{c}\text { ACEITE DE } \\
\text { OLIVA } \\
\text { VIRGEN }\end{array}$ & $\begin{array}{l}\text { ACEITE DE } \\
\text { GIRASOL } \\
\text { ALTO OLEICO }\end{array}$ \\
\hline Mujeres sanas & $13,9 \pm 2,6$ & $14,0 \pm 2,9$ & $5,9 \pm 2,6^{*}$ \\
\hline HT/normocolesterolémicas & $18,3 \pm 3,9$ & $13,2 \pm 1,6^{\star}$ & $6,9 \pm 3,2^{*}$ \\
\hline HT/hipercolesterolémicas & $20,3 \pm 6,9$ & $11,6 \pm 1,8^{*}$ & $8,4 \pm 3,9^{*}$ \\
\hline
\end{tabular}

Los valores se expresan como las medias (minutos) \pm desviación estándar ${ }^{*} \mathrm{p}<0,001$, con respecto a la dieta basal

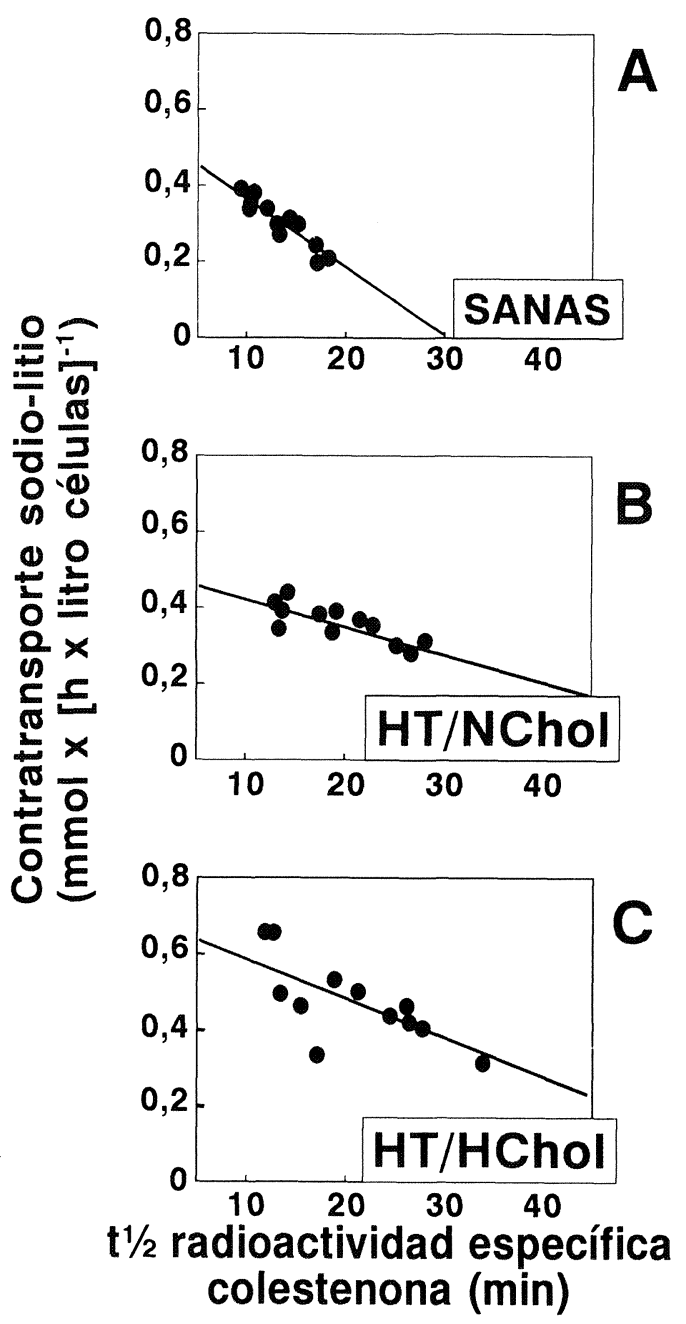

Figura 7

\section{Presión arterial (sistólica y diastólica)}

La presión arterial fue naturalmente superior (con significado estadístico) en pacientes hipertensas normocolesterolémicas (sistólica: $32 \%, p<0.01$; diastólica: $31 \%, p<0.01$ ) y pacientes hipertensas hipercolesterolémicas (sistólica: $34 \%, p<0.01$; diastólica: $27 \%, p<0.01$ ) que en mujeres sanas (sistólica: $122.3 \pm 8.1 \mathrm{~mm} \mathrm{Hg}$; diastólica: $72.4 \pm 5.3 \mathrm{~mm} \mathrm{Hg}$ ) (Tabla 4).

Tras la ingesta de aceite de oliva virgen con respecto a la dieta basal, los valores de presión arterial disminuyeron de manera significativa en los tres grupos experimentales: mujeres sanas (sistólica: $3 \%, p<0.05$; diastólica: $4 \%, p<0.05$ ), pacientes hipertensas normo- (sistólica: $6 \%, p<0.05$; diastólica: $11 \%, p<0.05$ ) e hiper- (sistólica: $6 \%, p<0.05$; diastólica: $7 \%, p<0.05$ ) colesterolémicas. Aunque los cambios producidos en las pacientes hipertensas fueron superiores a los de mujeres sanas, también disminuyó la presión arterial en éstas, indicando que el efecto hipotensor del aceite de oliva virgen no está exclusivamente asociado a la normalización de la actividad del contratransporte sodio-litio, y de la distribución y velocidad de movimiento del colesterol en las membranas. El aceite de girasol alto-oleico no tuvo efecto alguno sobre la presión arterial.

Uno de los mecanismos propuestos para explicar la reducción de la presión arterial es la incorporación de ácidos grasos a las membranas celulares del tejido vascular, regulando la actividad de enzimas, la reactividad vascular y la permeabilidad iónica (Engler, 1992) (Straznicky, 1993). No podemos descartar, por tanto, que la incorporación de ácidos grasos de la familia n-3 en la membrana de eritrocito de mujeres sanas y pacientes hipertensas puede ser responsable de éstos y otros fenómenos relacionados con la hipotensión que se observa tras la ingesta de 
aceite de oliva virgen. Probablemente, la normalización de los parámetros relacionados con la funcionalidad y estructura de las membranas biológicas sea incluso un paso previo a la acción derivada de la modificación en la composición en ácidos grasos de los fosfolípidos de membrana.

Tabla 4

Presión arterial (sistólica y diastólica) durante la dieta basal y tras las dietas enriquecidas con aceite de oliva virgen y aceite de girasol alto-oleico

\begin{tabular}{|c|c|c|c|}
\hline$\checkmark$ Presión arterial sistólica & BASAL & $\begin{array}{c}\text { ACEITE DE } \\
\text { OLIVA } \\
\text { VIRGEN }\end{array}$ & $\begin{array}{c}\text { ACEITE DE } \\
\text { GIRASOL } \\
\text { ALTO OLEICO }\end{array}$ \\
\hline Mujeres sanas & $122,3 \pm 8,10$ & $118,8 \pm 9,62^{*}$ & $122,5 \pm 8,75$ \\
\hline HT/normocolesterolémicas & $161,2 \pm 14,4$ & $151,2 \pm 10,3^{*}$ & $155,1 \pm 7,07$ \\
\hline HT/hipercolesterolémicas & $163,9 \pm 9,61$ & $156,7 \pm 8,54^{*}$ & $161,7 \pm 10,3$ \\
\hline \multicolumn{4}{|l|}{$\checkmark$ Presión arterial diastólica } \\
\hline Mujeres sanas & $72,4 \pm 5,3$ & $69,1 \pm 5,6^{*}$ & $71,4 \pm 5,0$ \\
\hline HT/normocolesterolémicas & $94,9 \pm 5,0$ & $84,9 \pm 8,1^{*}$ & $90,1 \pm 8,7$ \\
\hline HT/hipercolesterolémicas & $92,0 \pm 3,1$ & $86,1 \pm 8,9^{*}$ & $91,5 \pm 6,4$ \\
\hline
\end{tabular}

Los valores se expresan como las medias $(\mathrm{mm} \mathrm{Hg}) \pm$ desviación estándar

${ }^{*} \mathrm{p}<0,05$, con respecto a la dieta basal

\section{CONCLUSIONES Y RECOMENDACIONES}

Globalmente, los resultados de este estudio nos muestran que el aceite de oliva virgen es un alimento con propiedades cardiosaludables. Esta intervención dietética ha sido contrastada con otro aceite vegetal monoinsaturado (aceite de girasol alto-oleico) y analizando su efectos sobre factores de riesgo vascular (de máxima importancia): hipercolesterolemia e hipertensión arterial.

Se comprobó el concepto de «efectividad» de ambos aceites para aumentar las lipoproteínas de alta densidad (HDL) (antiaterogénica) en el plasma sanguíneo de la población estudiada (Ruiz-Gutiérrez, 1997), lo cual indica que mediante una dieta enriquecida en grasas monoinsaturadas es posible controlar y/o prevenir parcialmente las enfermedades cardiovasculares asociadas con dislipemias.

Lo más importante es que el aceite de oliva virgen fue el único capaz de reducir de manera significativa la presión arterial (sistólica y diastólica) de pacientes hipertensas normocolesterolémicas y pacientes hipertensas hipercolesterolémicas. Este efecto hipotensor del aceite de oliva virgen fue concordante con la normalización de parámetros celulares que inciden en el desarrollo de la Patología
Cardiovascular: actividad del contratransporte sodio-litio, distribución asimétrica del colesterol y su velocidad de movimiento transmembrana. Por lo tanto, la ingesta de aceite de oliva virgen puede contribuir al equilibrio iónico del sodio intracelular y a mejorar el transporte reverso del colesterol.

Nuevos estudios serán necesarios para determinar el(los) componente(s) del aceite de oliva virgen responsable(s) de los cambios observados. Es evidente que una mejora de la calidad de vida mediante un aceite de oliva virgen de calidad contribuye a potenciar su consumo, el sector productivo e industrial, y reducir costes socio-económicos derivados del inicio y complicaciones de las enfermedades cardiovasculares.

\section{AGRADECIMIENTOS}

Al Servicio de Medicina Interna del Hospital Universitario Virgen del Rocío de Sevilla, y a la Comisión Interministerial de Ciencia y Tecnología (CICYT) por el apoyo económico al presente estudio mediante el Proyecto-Al I 96-0456. 


\section{BIBLIOGRAFÍA}

Applegate, K. R. y Glomset, J. A. (1986).- “Computer-based modeling of the conformation and packing properties of docosahexaenoic acid".- J. Lipid Res. 27: 658680.

Aranda, P. y Villar, J. (1993).-—Estudio epidemiológico andaluz sobre factores de riesgo vascular».- Servicio Andaluz de Salud, Sevilla.

Aviv, A. (1988a).- - The link between cytosolic $\mathrm{Ca}^{2+}$ and the $\mathrm{Na}^{+}-\mathrm{H}^{+}$antiport: a unifying factor for essential hypertension".- J. Hypertens. 6: 685-691.

Aviv, A. y Liune, A. (1988b). - "The $\mathrm{Na}^{+} / \mathrm{H}^{+}$antiport, cytosolic free $\mathrm{Ca}^{2+}$, and essential hypertension: an hypothesis".Am. J. Hypertens. 1: 410-413.

Blaustein, M. P. (1984). - «Sodium Transport and Hypertension. Where are we going?».- Hypertension. 6: 445-453.

Brasaemle, D. L., Robertson, A. D. y Attie, A. D. (1988)."Transbilayer movement of cholesterol in the human erythrocyte membrane».- J. Lipid Res. 29: 481-488.

Brolin, S. E. (1983). — «Adenylate Kinase» en «Methods of Enzymatic Analysis" Vol. 3, p. 545.- H. E. Bergmeyer, (Ed.). - Weinheim: VCH.

Canessa, M., Morgan, K. y Semplicini, A. (1988)._-"Genetic differences in lithium-sodium exchange and regulation of the sodium-hydrogen exchanger in essentia hypertension".- J. Cardiovasc. Pharmacol. 12: 592598.

Canessa, M., Morgan, K., Goldszer, R., Moore, T. J. y Spalvins, A. (1991).- - Kinetic abnormalities of the red blood cell sodium-proton exchanger in hypertensive patients".- Hypertension. 17: 340-348.

Coca, A. (1993). - "Sodio e Hipertensión Arterial» en "Alteraciones Fisiopatológicas en el Hipertenso. Aplicaciones Terapéuticas» p. 109.- P. Aranda, (Ed.).Liga Española para la Lucha contra la Hipertensión Arterial, Madrid.

Corrocher, R., Pagnan, A., Ambrosio, G. B., et al. (1992).«Effects induced by olive oil-rich diet on erythrocytes membrane lipids and sodium-potassium transports in post-menopausal hypertensive women».-J. Endocrinol. Invest. 15: 369-376.

Engler, M. M., Engler, M. B., Erickson, S. K. y Paul, S. M. (1992)._- ¿Dietary gamma-linolenic acid lowers blood pressure and alters aortic reactivity and cholesterol metabolism in hypertension».- J. Hypertens. 10: 1197-1204.

Knapp, H. R., Hullin, F. y Salem, N. (1994).- -Asymmetric incorporation of dietary $n-3$ fatty acids into membrane aminophospholipids of human erythrocytes".- J. Lipid Res. 35: 1283-1291.

Lange, Y. (1992). - «Tracking cell cholesterol with cholesterol oxidase».- J. Lipid Res. 33: 315-321.

Liscum, L. y Faust, J. R. (1994)._- «Compartimentation of cholesterol within the cell».- Curr. Opin. Lipidol. 5: 221-226.

Muriana, F. J. G., García-Donas, M. A., Villar, J. y Ruiz-Gutiérrez, V. (1994).- - «Distribution of erythrocyte membrane cholesterol in human essential hypertension".J. Hypertens. 12: 1383-1386.

Muriana, F. J. G., Montilla, C., Villar, J. y Ruiz-Gutiérrez, V. (1995). - «Transbilayer movement of erythrocyte membrane cholesterol in human essential hypertension».J. Hypertens. 13: 619-623.

Muriana, F. J. G., Montilla, C., Stiefel, P., Villar, J. y RuizGutiérrez, V. (1996a). - «The rate of transbilayer movement of erythrocyte membrane cholesterol is correlated with sodium-lithium countertransport».Life Sci. 59: 1945-1949.

Muriana, F. J. G., Villar, J. y Ruiz-Gutiérrez, V. (1996b).«Erythrocyte membrane cholesterol distribution in patients with untreated essential hypertension: correlation with sodium-lithium countertransport».- J. Hypertens. 14: 443-446.

Muriana, F. J. G., Alonso, A., Villar, J. y Ruiz-Gutiérrez, V. (1996c).- «Validity of studies on distribution and transbilayer movement of erythrocyte membrane cholesterol».- J. Hypertens. 14: 1379-1380.

Nemecz, G., Fontaine, R. N. y Schroeder, F. (1988a).— «A fluorescence and radiolabel study of sterol exchange between membranes".- Biochim. Biophys. Acta 943: 511-52.

Nemecz, G. y Schroeder, F. (1988b).-«Membrane cholesterol heterogeneity and exchange studied using dehydroergosterol'».- Biochemistry 27: 7740-7749.

Otero, M. L., Pinilla, M. C. F. y Candela, C. G. (1990)."Hipertensión y dieta».-Internacional de Ediciones y Publicaciones, Madrid.

Ronquist, G., Frithz, G., Gunnarsson, K. y Arvidson, G. (1992). - «Decreased erythrocyte cholesterol/phospholipid ratio in untreated patients with essential hypertension».J. Intern. Med. 232: 247-251.

Rothblat, G. H., Mahlberg, F. H., Johnson, W. J. y Phillips, M. C. (1992).— -Apolipoproteins, membrane cholesterol domains, and the regulation of cholesterol efflux».- $J$. Lipid Res. 33: 1091-1097.

Ruiz-Gutiérrez, V., Muriana, F. J. G. y Villar, J. (1998).«El aceite de oliva virgen y las enfermedades cardiovasculares. Perfil lipídico en plasma y composición lipídica de la membrana de eritrocito humano".- Grasas y Aceites 49: 9-29.

Rutherford, P. A., Thomas, T. H. y Wilkinson, R. (1992).«Erythrocyte sodium-lithium countertransport: Clinically useful, pathophysiologically instructive or just phenomenology?». - Clin. Sci. 82: 341-352.

Schroeder, F., Jefferson, J. R., Kier, A. B., et al. (1991)."Membrane cholesterol dynamics: cholesterol domains and kinetic pools».- Proc. Soc. Exp. Biol. Med. 196: 235-252

Schroeder, F. y Nemecz, G. (1990).— «Transmembrane Cholesterol Distribution" en "Advances in Cholestero Research" p. 47. - Telford Press, Londres.

Shouffani, A. y Kanner, B. I. (1990).- "Cholesterol is required for the reconstitution of the sodium- and chloride-coupled, gamma-aminobutyric acid transporter from rat brain».- J. Biol. Chem. 265: 6002-6008.

Straznicky, N. S., Louis, W. J., MeGrade, P. y Howes, L. G. (1993). - «The effects of dietary lipid modification on blood pressure, cardiovascular reactivity and sympathetic activity in men".- J. Hypertens. 11: 427-437.

Tamargo, J. (1993).- - «Arteriosclerosis e hipertensión» en "Alteraciones Fisiopatológicas en el Hipertenso. Aplicaciones Terapéuticas» p. 151.- P. Aranda, (Ed.).Liga Española para la Lucha contra la Hipertensión Arterial, Madrid.

Vassault, A. (1983).- “Lactate Dehydrogenase» en «Methods of Enzymatic Analysis» Vol. 3, p. 118.- - H. E. Bergmeyer, (Ed.).- Weinheim: $\mathrm{VCH}$. 